ФГБУ «НМИЦ им. В.А. Алмазова» Минздрава России, Санкт-Петербург

Сахарный диабет (СД) 2 типа является одним из наиболее распространенных заболеваний как в мире, так и в Российской Федерации, при котором хронические осложнения, развивающиеся в условиях гипергликемии, представляют серьезную социально-экономическую проблему. Помимо классических осложнений СД, таких как диабетическая ретинопатия, нефропатия и полинейропатия, в последнее время обращает на себя внимание наличие повышенного риска переломов у больных СД 2 типа даже при нормальных показателях минеральной плотности кости, получившее название «диапороз», или «диабетопороз», и рассматривающееся как еще одно осложнение СД. Известно, что остеопороз, так же как и СД 2 типа, является широко распространенной патологией и встречается чаще у лиц старшей возрастной группы, что делает их сочетание у одного пациента весьма вероятным. Принимая во внимание высокую социальную значимость и инвалидизацию вследствие остеопоротических переломов и хронических осложнений СД 2 типа, становится необходимым продолжение изучения физиологических и биохимических основ формирования и ремоделирования костной ткани, а также патогенетических механизмов остеопороза в условиях хронической гипергликемии. Одним из довольно новых факторов, который, по мнению экспертов, может представлять связующее звено между нарушением углеводного обмена и костным ремоделированием, является остеокальцин, концентрация которого находится в зависимости от обеспечения витамином К2, который сегодня рассматривается как потенциальный агент для профилактики остеопоротических переломов. В данной обзорной статье будет освещена актуальная информация о влиянии витамина К2 на состояние костной ткани и возможности использования данного нутриента в клинической практике с фокусом на больных СД.

КЛЮЧЕВЫЕ СЛОВА: сахарный диабет; витамин К2; костная ткань; остеопороз.

\title{
BONE METABOLISIM IN DIABETES MELLITUS: FOCUS ON VITAMIN K2
}

\author{
(c) Fyodor M. Radugin, Tatiana L. Karonova
}

Almazov National Medical Research Centre, Saint-Petersburg

Diabetes mellitus (DM) type 2 is reported to be among the most common diseases both worldwide and in Russian Federation, whereby chronic implications that develop under hyperglycemia pose a serious socioeconomic problem. Besides of classic microvascular complications like diabetic retinopathy, nephropathy and polyneuropathy it's recently worthy of note the presence of high fracture risk in DM type 2 even in normal bone mass density, known as "diaporosis" or "diabetoporosis" considered as another DM complication. It is well known that osteoporosis is reported to be widespread disease as same as DM type 2 and likewise is a widely abundant in older age that makes their combination is really possible in a single patient. Taking into account high social value and disability due to osteoporotic fractures and chronic DM type 2 complications it becomes necessary to continue physiologic and biochemical basis investigations of bone formation and remodeling and pathogenesis of osteoporosis development in the context of chronic hyperglycemia. One of the fairly new factors which the experts believe it is link between impaired glucose metabolism and bone remodeling is osteocalcin which concentration is dependent on vitamin $\mathrm{K} 2$ maintenance considered as a potential agent in osteoporotic fractures prevention. In this review current information on vitamin K2 influence on bone status and possibility of using this nutrient in clinical practice in the focus on patients with DM will be covered.

KEYWORDS: diabetes mellitus; vitamin k2; bone; osteoporosis.

\section{ВИТАМИН К: ОТКРЫТИЕ, ИЗОФОРМЫ, ИСТОЧНИКИ}

Открытие витамина К связывают с именем датского ученого Henrik Dam, изучавшего в 1929 г. биологические эффекты холестерина. Экспериментальным путем он подтвердил существование жирорастворимого нутриента, влияющего на процесс коагуляции. Впоследствии Henrik Dam смог идентифицировать это вещество как витамин K («koagulation» — дат.) [1]. А позже, в 30-е гг. прошлого столетия, Edward Doisy определил молекулярную структуру витамина $\mathrm{K}_{1}$ - филохинона [1]. В дальнейшем был выделен другой витамер - менахинон, также известный как витамин $\mathrm{K}_{2}[1,2]$. Длительное время предполагалось, что биологические эффекты витаминов $\mathrm{K}_{1}$ и $\mathrm{K}_{2}$ идентичны и заключаются в прокоагулянтном действии [3]. Однако в течение последних 30 лет интерес научного сообщества все чаще вызывает проблема биологических эффектов витамеров $\mathrm{K}_{1}$ и $\mathrm{K}_{2}$, а также скрытого 
дефицита витамина $\mathrm{K}_{2}$, который является в настоящее время широко распространенным состоянием [1, 2, 4-6].

Витамин К существует в человеческом организме в нескольких разновидностях, витамерах, и необходим организму для синтеза различных кальций-связывающих белков. Витамин $\mathrm{K}_{1}$ - филохинон - близок к хлорофиллу и содержится в продуктах растительного происхождения: зеленые листовые овощи, капуста, шпинат, щавель. Филохинон поступает из двенадцатиперстной кишки в кровь, где находится в течение 2-3 ч $[1,4,5]$. Витамин $\mathrm{K}_{2}$ - менахинон - содержится в ферментированных сырах, говяжьей печени, сливочном масле, а также синтезируется из метаболитов бактерий вида E. coli и Bacteroides fragilis, являющихся представителями нормофлоры тонкой кишки человека и многих животных $[4,7]$. Микробный синтез витамина $\mathrm{K}_{2}$ стимулируется при недостатке филохинона в диете и способен его полностью компенсировать, однако недостаточность менахинона при сниженной активности микрофлоры плохо корригируется диетическими мероприятиями [1, 2]. Следовательно, подавление микрофлоры тонкой кишки или ее избыточный рост приводит к количественному нарушению синтеза витамина К [1, 3, 5-7].

Таким образом, витамин К представляет собой группу органических соединений, объединенных на основании структурного принципа, но значительно отличающихся функционально [1, 6].

\section{БИОЛОГИЧЕСКИЕ ЭФФЕКТЫ ВИТАМИНА К}

Витамин К представляет собой кофермент для витамин-К-зависимой гамма-глутаматкарбоксилазы, осуществляющей посттрансляционное карбоксилирование глутаминовой кислоты в полипептидных цепях ряда белков [8]. В карбоксилированной форме витамин-Кзависимые белки лучше секретируются клетками, накапливаются в тканях и выполняют свои биологические функции [9]. Основной точкой приложения витамина К в организме является система свертывания, где он выполняет роль ко-фактора и участвует в активации как факторов свертывания (протромбин (II), проконвертин (VII), антигемофильный глобулин B (IX), фактор СтюартаПрауэра (X)), так и антипротеаз плазмы С и S [1]. С другой стороны, другим важным витамин-К-зависимым белком является остеокальцин (bone-Gla-protein, Osteocalcin, OC) [1, 8-10], представляющий собой неколлагеновый белок экстрацеллюлярного матрикса костной ткани [8]. ОС синтезируется преимущественно остеобластами, составляет $25 \%$ всей неколлагеновой части органического матрикса кости или 2\% массы костной ткани в целом $[8,10]$. Необходимое условие нормального функционирования ОС - карбоксилирование в 17, 21 и 24 положениях с помощью фермента гамма-глутамилкарбоксилазы, кофактором которого является витамин К $[2,9]$. Именно остатки гамма-карбоксиглутаминовой кислоты, появившиеся в структуре ОС в ходе реакции карбоксилирования, притягивают на себя 3 иона кальция и укладывают их в структуру гидроксиапатита, который является основной составляющей костной ткани и обеспечивает ее минеральную плотность [2, 9]. Полностью карбоксилированный ОС обладает наибольшим сродством к костной ткани и практически не выходит в системный кровоток, что способствует минерализации костей и адекватному формированию основного вещества костной ткани [8]. Важно отметить, что при недостатке витамина К образуются менее карбоксилированные формы ОС: «не полностью карбоксилированный ОС» - карбоксилирование производится только в 17 и 24 положениях, реже в 21 и 24 положениях; «недокарбоксилированный ОС»карбоксилирование только по 17 положению [8]. При выраженном дефиците витамина К часть ОС не карбоксилируется вовсе. Все вышеперечисленные менее карбоксилированные формы ОС обладают меньшим сродством к костной ткани, а также легко проникают в системный кровоток, где обладают собственной биологической активностью: влияют на развитие головного мозга, на фертильность у мужчин, а также участвуют в углеводном и энергетическом обмене [9]. Помимо формирования основного вещества костной ткани, витамин К участвует в регуляции популяции остеокластов, поддерживая их запрограммированную гибель, и тем самым предотвращает избыточную резорбцию костной ткани $[2,6,8,9,10]$.

Другим значимым витамин К-зависимым белком является матриксный белок Gla (matrix Gla protein, MGP), который вырабатывается хондроцитами и клетками гладких мышц сосудов и предотвращает отложение кальция в сосудах $[2,8,9]$. Карбоксилированная форма данного белка играет ключевую роль в ингибировании роста кристаллов кальция в мягких тканях $[2,8,9]$. Предполагается, что супрессивное действие MGP происходит на уровне транскрипции генов прокальцификации, подавления остеохондрогенной дифференцировки сосудистых гладкомышечных клеток, а также осуществляется посредством прямого торможения роста кристаллов кальция [2].

Таким образом, витамин К посредством гамма-карбоксилирования MGP может выступать фактором ангиопротекции, что может служить гарантией защиты стенок сосудов от избыточной патологической минерализации. Кроме того, сам уровень MGP может служить биомаркером активного процесса сосудистой кальцификации $[2,8,9]$. Из этого следует, что биологические эффекты витамина К не ограничиваются влиянием на систему свертывания и противосвертывания, как это было принято ранее, а имеют куда более глобальное значение, включая минерализацию костной ткани посредством увеличения концентрации карбоксилированного ОС [2, 8-10].

\section{ВЛИЯНИЕ ВИТАМИНА К}

Долгое время в качестве витамина, оказывающего потенциально профилактическое влияние в отношении переломов, рассматривали витамин D. Несмотря на положительные эффекты витамина D на кость и мышечную ткань, рандомизированные клинические исследования не показали снижение риска падений и переломов на фоне приема больших доз витамина D [11]. Учитывая вышеперечисленные биологические эффекты витамина К, а именно его участие в карбоксилировании ОС и минерализации кости, в последние годы витамин $\mathrm{K}_{2}$ стал рассматриваться в качестве дополнительного нутриента для снижения риска переломов и падений. На сегодняшний день опубликованы результаты небольшого количества экспериментальных и клинических исследований, 
демонстрирующих механизм и роль витамина $\mathrm{K}_{2}$ в метаболизме костной ткани.

Так, в исследовании in vitro, проведенномподруководством S. Urayama, витамин $\mathrm{K}_{2}$ стимулировал пролиферацию стволовых клеток костного мозга, дифференцировку остеобластов, защищал их от апоптоза и ингибировал дифференцировку адипоцитов [12]. Важно отметить, что такой эффект не был обнаружен при применении витамина $\mathrm{K}_{1}$ [12]. Исследователями отмечено, что увеличение числа остеоцитов на фоне повышения популяции остеобластов при приеме витамина $\mathrm{K}_{2}$ улучшает состояние лакунарной системы и снижает порозность кости, а также снижается экспрессия лиганда рецептора активатора нуклеарного фактора кB (RANKL) и активируется апоптоз остеокластов [13] как напрямую, так и косвенно, в том числе через увеличение секреции остеопротегерина (ОПГ) [14]. Таким образом, результаты данных исследований позволили сделать вывод об анаболическом эффекте витамина $\mathrm{K}_{2}$ на костное ремоделирование. Дополнительно было показано, что при добавлении витамина $\mathrm{K}_{2}$ происходит ингибирование сигнального пути NF-кB, что проявляется в снижении экспрессии мРНК интерлейкина-6 (ИЛ-6), фактора некроза опухоли-а (ФНО-а) и ИЛ-1 $\beta$ в липополисахарид-обработанных клетках мышей [15] и снижении ИЛ-6 в липополисахарид-обработанных человеческих ТНР-1 клетках и фибробластах [16], что указывает на противовоспалительные эффекты данного нутриента. Интересно отметить, что снижение ИЛ-6 наблюдается при использовании всех изоформ витамина K, однако наибольшая эффективность присуща $\mathrm{K}_{3}$ [16]. Интересное исследование норвежских ученых показало положительный эффект добавления витамина $\mathrm{K}_{2}$ в комбинации с витамином D на прочность трехмерной модели костной ткани, созданной из человеческих остеобластов [17]. Благоприятное влияние на активацию остеобластов и восстановление кости было отмечено и при исследовании комбинации $\mathrm{K}_{2}$ с рекомбинантным человеческим паратиреоидным гормоном (ПТГ) [18, 19].

Использование витамина $\mathrm{K}_{2}$ с лечебной целью у животных с остеопорозом, индуцированным овариэктомией или орхидэктомией [20], приемом глюкокортикоидов [21], удалением седалищного нерва [22] или дефицитом в диете кальция и магния [23], было продемонстрировано в различных работах. Результаты этих исследований показали, что данная терапия ассоциирована со снижением костной резорбции и/или повышением активности остеобластов. Витамин $\mathrm{K}_{2}$ предотвращал снижение минеральной плотности костной ткани (МПКТ) в бедренной кости, потерю трабекулярной и кортикальной костной ткани и резорбцию в поясничных позвонках. Добавление витамина D и кальция к витамину $\mathrm{K}_{2}$ ингибировало остеокластогенез у крыс с овариэктомией [24]. Также применение комбинированной терапии витамина $\mathrm{K}_{2}$ с витамином $\mathrm{D}$ [25] или бисфосфонатами [26] показало дополнительный протективный эффект в отношении остеопороза по сравнению с монотерапией витамином $\mathrm{K}_{2}$. Тем не менее не все исследования показали позитивный эффект витамина $\mathrm{K}_{2}$ на кость, в частности при остеопорозе, индуцированном овариэктомией $[20,27]$.

Результаты же клинических исследований остаются весьма ограниченными. Так, исследование по оценке риска переломов в зависимости от потребления витамина $\mathrm{K}_{2}$ с пищей, проведенное среди японских женщин в постменопаузе, показало, что его низкое содержание ассоциировано с повышенным риском переломов [28]. Большинство интервенционных исследований показало протективный эффект витамина К на состояние костной ткани и снижение риска переломов у здоровых женщин в постменопаузе и у женщин с постменопаузальным остеопорозом [29-32]. Метаанализ 13 исследований, в которых была использована изоформа витамина К в виде MK-4, показал протективный эффект как в виде повышения МПКТ, так и снижения риска переломов бедра, вертебральных и невертебральных переломов [33]. Необходимо отметить тот факт, что в ряде исследований использовались большие дозы витамина $\mathrm{K}_{2}$ (45 мг в сутки), что и позволило авторам получить положительные результаты. Следует отметить, что хотя обычная суточная потребность витамина К составляет 250-300 мкг, использование дозы, превышающей норму, не приводит к появлению побочных эффектов на протяжении 2 лет [29]. Несмотря на вышеописанные данные, крупные нерандомизированные и рандомизированные плацебо-контролируемые исследования не доказали эффективность применения витамина $\mathrm{K}_{2}$ в профилактике остеопоротических переломов [34-36]. Так, метаанализ рандомизированных контролируемых исследований с 6759 женщинами в постменопаузе без остеопороза не показал влияния витамина $\mathrm{K}_{2}$ на костную ткань [37]. Важно отметить, что при оценке уровня изоформы витамина $\mathrm{K}_{2}-$ менахинона-7 у женщин с остеопорозом с/без переломов не выявлено разницы в показателях по результатам исследования, проведенного Т. Horiuchi и соавт. [30]. Лимитирующим фактором таких исследований нередко являлось отсутствие возможности определения изоформы менахинона-4 в крови как формы, усваиваемой исключительно из пищи [38]. Таким образом, в настоящий момент не получено достаточно данных, подтверждающих преимущество приема витамина $\mathrm{K}_{2}$ в монотерапии, так как небольшие исследования показывают позитивный эффект такой терапии, а крупные исследования демонстрируют отсутствие эффекта от данной терапии [34-37, 39]. Вместе с тем исследования, где использовалась комбинация витамина $\mathrm{K}_{2}$ и витамина D, показали позитивное влияние такой терапии на показатели МПКТ поясничного отдела позвоночника при первичном и постменопаузальном остеопорозе у женщин [40], в том числе и в сравнении с монотерапией витамином $\mathrm{K}_{2}$ [41]. Эта комбинация предотвращала потерю костной ткани у пациентов, получающих глюкокортикостероиды по поводу хронического гломерулонефрита [42]. Также в отношении сохранения МПКТ поясничного отдела позвоночника у женщин в постменопаузе эффективность показала и тройная комбинация витамина $\mathrm{K}_{2}$ в дозе 100 мкг в день, кальция в дозе 800 мг в день и витамина D в дозе 10 мкг (400 ME) в день [43]. Однако в недавнем рандомизированном контролируемом исследовании популяции постменопаузальных китайских женщин средних лет и в пожилом возрасте дополнительное добавление к витамину $\mathrm{K}_{2}$ в дозе 90 мкг в день препаратов кальция (500 мг в день) и витамина D (в дозе $400 \mathrm{ME}$ 
в день) не показало улучшения эффекта в сравнении с монотерапией витамином $\mathrm{K}_{2}$ [44]. Единичные исследования применения витамина $\mathrm{K}_{2}$ с антирезорбтивными препаратами, в частности с бисфосфонатами, также продемонстрировали неоднозначные результаты: так, комбинация с алендронатом была ассоциирована с повышением МПКТ в бедренной кости у женщин с постменопаузальным остеопорозом [45], в то время как комбинированная терапия с ризедронатом не показала эффективности по сравнению с монотерапией ризедронатом [46].

Таким образом, целесообразность монотерапии или комбинированной терапии витамином $\mathrm{K}_{2}$ в настоящий момент остается весьма дискутабельной и требует дальнейших исследований.

\section{САХАРНЫЙ ДИАБЕТ И КОСТНАЯ ТКАНЬ}

Известно, что сахарный диабет (СД) является независимым фактором риска остеопороза [47]: у таких больных он выше, чем у пациентов без СД, особенно при СД 1 типа, когда он в 3-6 раз выше, чем у лиц соответствующего возраста без диабета [48]. У пациентов с СД 1 типа обычно имеет место снижение МПКТ [49], в то время как при СД 2 типа МПКТ не снижена и даже повышена $[50,51]$, однако имеет место низкое качество костной ткани $[52,53]$. Возможно, данное обстоятельство объясняет, почему риск остеопоротических переломов выше у больных СД 1 типа, чем у больных СД 2 типа. При СД отмечается снижение трабекулярного костного индекса (ТКИ) [54, 55], последнее время широко применяющегося для прогнозирования переломов при СД $[56,57]$. ТКИ обратно пропорционален уровням гликированного гемоглобина $\left(\mathrm{HbA}_{1 c}\right)$, глюкозы плазмы и инсулина натощак [58]. СД ассоциирован с такими состояниями, как диабетическая периферическая полинейропатия, ретинопатия, гипогликемические эпизоды, нарушение равновесия, заболевания сердца и нейромышечные нарушения, что увеличивает риск падений и, соответственно, переломов [53, 55, 59].

У больных СД отмечается нарушение ремоделирования кости и формируется хрупкая костная ткань [55]. Хроническая гипергликемия нарушает баланс между остеокластами и остеобластами, воздействуя на дифференцировку мезенхимальных прекурсоров, локальные ростовые факторы и нарушая ответ на механический стресс. Отмечаются микроархитектурные нарушения в кости, которые возникают за счет ограничения дифференцировки остеобластов и усиленного адипогенеза в костном мозге, в том числе из-за повышения активности PPRY2, peзистина и аР2 [60]. Дополнительно к этому имеются указания на изменение активности генов и синтеза транскрипционных факторов, отвечающих как за остеобластогенез (Runx2, DIx5, Osx, ALP и других), так и за остеокластогенез (ОПГ и MMP9) [55] при высоком уровне глюкозы плазмы крови. Также у больных СД имеется снижение RANKL, взаимодействие которого со специфическим рецептором (RANK) и ОПГ связано с дифференцировкой и активацией остеокластов [58], и снижение уровня которого ассоциировано с нарушением костного ремоделирования в виде нарушения обновления костной ткани на фоне нарушения остеокластогенеза.
Дополнительно, к важным этиопатогенетическим компонентам формирования так называемой «диабетической кости» можно отнести и накопление конечных продуктов гликирования (КПГ), оказывающих негативное влияние на микроархитектуру кости и нарушающих клеточную биологию костной ткани [60]. КПГ влияют на микроархитектуру кости и нарушают клеточную биологию костной ткани. Аккумулирование КПГ и неферментативное гликозилирование напрямую воздействуют на экстрацеллюлярный костный матрикс за счет избыточного образования поперечных сшивок фибрилл коллагена I типа, повышая хрупкость кости и ухудшая ее биомеханические характеристики. Также КПГ индуцируют воспаление за счет связывания с рецепторами КПГ (РКПГ). Активация РКПГ инициирует каскад митоген-активируемой протеинкиназы, приводя к активации ядерного фактора кB (NF-кB) и продукции ФНО-а, ИЛ-1 $\beta$, ИЛ-6 и активных форм кислорода [61]. Таким образом, происходят активация остеокластов, апоптоз остеобластов, ограничение пролиферации мезенхимальных стволовых клеток и нарушение их дифференцировки в остеобласты [62]. Дополнительно при СД нарушается и другой сигнальный путь - Wnt (от англ. wingless - бескрылый). Так, оказывая влияние на остеоциты, КПГ повышают уровень склеростина. Склеростин - белок, ингибирующий сигнальный путь Wnt/ß-катенин и участвующий в пролиферации, дифференцировке мезенхимальных клеток и стимуляции апоптоза остеобластов [63]. Отмечают, что повышенный уровень склеростина зарегистрирован как у больных СД 1 типа, так и у больных СД 2 типа [64]. Более того, уровень склеростина у пациентов с СД 2 типа напрямую ассоциирован с повышением риска переломов [65]. Необходимо отметить и тот факт, что при СД 2 типа, несмотря на часто встречаемый дефицит витамина D, наблюдается более низкий уровень ПТГ, ОС, С-концевого телопептида коллагена 1 типа, $\mathrm{N}$-телопептида коллагена 1 типа и N-терминального пропептида проколлагена 1 типа по сравнению лицами без СД 2 типа [66], что свидетельствует о снижении скорости метаболизма костной ткани, наблюдающемся у данной популяции [47].

Дополнительно результаты проведенного метаанализа, включившего данные 22 исследований, показали, что концентрация ОС значимо ниже у больных СД [67]. Так, уровни как карбоксилированного, так и некарбоксилированного ОС у больных СД 2 типа негативно коррелировали с уровнем глюкозы крови натощак и степенью инсулинорезистентности [8]. Есть данные о липолитическом эффекте, усилении $\beta$-клеточной пролиферации, улучшении секреции инсулина, повышении чувствительности мышечной ткани к инсулину и увеличении ее массы при применении ОС $[2,8,9]$. Все эти результаты позволяют рассматривать ОС как потенциальную терапевтическую мишень у больных СД с целью уменьшения негативного влияния гипергликемии на костную ткань и профилактики переломов.

Анализируя влияние сахароснижающих препаратов на костную ткань, эксперты также приходят к мнению о наличии у ряда препаратов негативного влияния на увеличение риска как падений, так и остеопоротических переломов, что необходимо учитывать при 
выборе терапии у больных СД 2 типа. К таким препаратам сегодня можно отнести тиазолидиндионы (росиглитазон и пиоглитазон), на фоне терапии которыми при активации PPRy-рецепторов наблюдается перераспределение дифференцировки мезенхимальных клеток в пользу адипогенеза [68], а также ингибиторы натрий-глюкозного ко-транспортера 2 типа, в частности канаглифлозин, применение которых ассоциировано с повышением фактора роста фибробластов 23, снижением МПКТ и повышением риска переломов [69]. Однако требуется проведение дальнейших длительных проспективных сравнительных исследований для уточнения характера влияния данных препаратов на костную ткань $[68,70,71]$.

Таким образом, негативное влияние на костную ткань у больных СД обусловлено разными факторами, ассоциированными с данным заболеванием: хроническая гипергликемия, хронические осложнения СД, применение некоторых антигипергликемических лекарственных средств, способными как нарушать костное ремоделирование, так и напрямую негативно оказывать влияние на микроархитектуру кости и клеточную биологию костной ткани.

\section{ВЛИЯНИЕ ВИТАМИНА $K_{2}$ НА КОСТНУЮ ТКАНЬ У БОЛЬНЫХ СД}

Несмотря на то что применение витамина $\mathrm{K}_{2}$ у больных СД имеет определенные преимущества [72] и потенциальное позитивное воздействие на костную ткань, исходя из патогенетических механизмов влияния хронической гипергликемии на состояние кости у таких пациентов [47], существует довольно ограниченное количество работ, посвященных данной проблеме. Результаты исследований in vivo показали, что витамин $\mathrm{K}_{2}$ может улучшать качество кости при СД за счет повышения уровня карбоксилированного ОС [73]. Исследование на первичных остеобластах мышей с СД показало усиление анаболизма остеобластов при комбинированной терапии витамина $\mathrm{K}_{2}$ с кальцитриолом - активной формой витамина D [74]. В исследовании на крысах со стрептозоцин-индуцированным СД 1 типа было показано, что применение витамина $\mathrm{K}_{2}$ предотвращает остеопению в губчатых костях без значимого влияния на параметры костной резорбции, но незначимо повышает кортикальную костную массу [75]. Было предположено, что ухудшение состояния кости у животных с СД ассоциировано с дефектом карбоксилирования ОС с участием витамина $\mathrm{K}_{2}$. Действительно, в кросс-секционном исследовании с 85 пожилыми женщинами с СД 2 типа было выявлено значимое повышение уровня некарбоксилированного ОС у пациенток с остеопорозом по сравнению с пациентками без остеопороза. Корреляция между Z-критерием и логарифмическими уровнями некарбоксилированного/интактного ОС при СД 2 типа показала негативный тренд $(p=0,07)$, а у больных СД 2 типа с остеопорозом эта корреляция была значимой $(r=-0,61 ; P<0,05)$. Также это соотношение показало негативную корреляцию с уровнем MK-7 ( $r=-0,50$; $\mathrm{P}=0,001)$ [76]. Как следствие, было предположено, что снижение МПКТ в поясничном отделе позвоночника у больных с СД 2 типа было ассоциировано с дефек- том $\mathrm{Y}$-глутамилкарбоксилирования ОС витамином $\mathrm{K}_{2}$ что позволяет рассматривать назначение витамина $\mathrm{K}_{2}$ такой когорте больных с целью предотвращения снижения МПКТ. В последние годы были выполнены интервенционные исследования с участием пациентов с СД 2 типа. Рандомизированное двойное слепое исследование с участием 40 пациентов в возрасте от 30 до 70 лет показало, что применение витамина $\mathrm{K}_{2}$ в дозе 100 мкг в сутки в комбинации с витамином D в дозе $1000 \mathrm{ME}$ В сутки снижает индекс некарбоксилированный/ карбоксилированный ОС, при этом монотерапия витамином D снижала уровень некарбоксилированного OC, в то время как витамин $\mathrm{K}_{2}$ повышал уровень карбоксилированного ОС [77]. В свою очередь, ретроспективный анализ двойного слепого рандомизированного плацебо-контролируемого исследования показал, что ежедневный прием MK-7 в дозе 360 мкг в течение 6 мес не влияет на снижение МПКТ в поясничном отделе позвоночного столба [78].

Неоднородность полученных данных предполагает дальнейшее изучение применения витамина $\mathrm{K}_{2}$ в популяции больных СД с точки зрения его влияния на состояние костной ткани и профилактику переломов. Помимо экспериментальных работ, необходимы дальнейшие клинические исследования для оценки эффективности витамина $\mathrm{K}_{2}$.

\section{ЗАКЛЮЧЕНИЕ}

В настоящее время хорошо известен негативный эффект хронической гипергликемии на состояние костной ткани у больных СД. Имеющиеся на сегодняшний день данные углубляют знания исследователей и практикующих специалистов о механизмах такого воздействия. Это позволяет искать новые пути в профилактике костных нарушений у пациентов с СД. Витамин $\mathrm{K}_{2}$ показывает неоднозначные результаты в ходе клинических исследований среди больных СД, остеопорозом или практически здорового населения. Таким образом, роль витамина $\mathrm{K}_{2}$ в поддержании здоровья костной ткани и профилактике переломов, в частности у больных СД, требует уточнения и проведения дальнейших исследований. В настоящий момент нет убедительных данных, говорящих о благоприятном влиянии витамина $\mathrm{K}_{2}$ на состояние кости у больных диабетом и необходимости его приема с профилактической или лечебной целью.

\section{ДОПОЛНИТЕЛЬНАЯ ИНФОРМАЦИЯ}

Источники финансирования. Работа выполнена по инициативе авторов без привлечения финансирования.

Конфликт интересов. Авторы декларируют отсутствие явных и потенциальных конфликтов интересов, связанных с содержанием настоящей статьи.

Участие авторов. Радугин Ф.М. - анализ данных, написание статьи; Каронова Т.Л. - анализ данных, написание статьи, внесение существенной правки с целью повышения научной ценности статьи. Все авторы одобрили финальную версию статьи перед публикацией, выразили согласие нести ответственность за все аспекты работы, подразумевающую надлежащее изучение и решение вопросов, связанных с точностью или добросовестностью любой части работы. 


\section{СПИСОК ЛИТЕРАТУРЫ | REFERENCES}

1. Ferland G. The discovery of vitamin Kand its clinical applications. Ann Nutr Metab. 2012;61(3):213-218. doi: https://doi.org/10.1159/000343108

2. Gundberg CM, Lian JB, Booth SL. Vitamin K-dependent carboxylation of osteocalcin: friend or foe? Adv Nutr. 2012;3(2):149-157. doi: https://doi.org/10.3945/an.112.001834

3. Zurynski Y, Grover CJ, Jalaludin B, Elliott EJ. Vitamin K deficiency bleeding in Australian infants 1993-2017: an Australian Paediatric Surveillance Unit study. Arch Dis Child. 2020;105(5):433-438. doi: https://doi.org/10.1136/archdischild-2018-316424

4. Бельмер С.В. Роль кишечной микрофлоры в обеспечении организма фолиевой кислотой, витаминами В12 и K// Bonpoсb современной педиатрии. - 2005.- Т. 4. — №5. - С. 74-76. [Belmer SV. Rol kishechnoy mikroflory v obespechenii organizma folievoy kislotoy, vitaminami B12 i K. Voprosy sovremennoy pediatrii. 2005;4(5):74-76. (In Russ.)].

5. Palermo A, Tuccinardi D, D'Onofrio L, et al. Vitamin K and osteoporosis: Myth or reality? Metabolism. 2017;70:57-71. doi: https://doi.org/10.1016/j.metabol.2017.01.032

6. Beulens JW, Booth SL, van den Heuvel EG, et al. The role of menaquinones (vitamin $\mathrm{K}_{2}$ ) in human health. Br J Nutr. 2013;110(8):1357-1368. doi: https://doi.org/10.1017/S0007114513001013

7. Громова О.А., Торшин И.Ю., Гарасько Е.В., и др. Системный анализ взаимосвязей между метаболизмом витаминов микробиотой и выживанием позитивной микрофлоры ЖКТ // Экспериментальная и клиническая гастроэнтерология. 2013. — №2. - C. 28-36. [Gromova OA, Torshin IYu, Garas'ko EV. et al System analysis of the relationship between the metabolism of vitamins by micro-biota and the survival of the positive microflora of the digestive tract. Experimental and clinical gastroenterology. 2013;2:28-36 (In Russ.)].

8. Li J, Zhang H, Yang C, Li Y, Dai Z. An overview of osteocalcin progress. J Bone Miner Metab. 2016;34(4):367-379. doi: https://doi.org/10.1007/s00774-015-0734-7

9. Mizokami A, Kawakubo-Yasukochi T, Hirata M. Osteocalcin and its endocrine functions. Biochem Pharmacol. 2017;132:1-8. doi: https://doi.org/10.1016/j.bcp.2017.02.001

10. Панкратова Ю.В., Пигарова Е.А., Дзеранова Л.К. Витамин K-зависимые белки: остеокальцин, матриксный Gla-белок и их внекостные эффекты // Ожирение и метаболизм. - 2013. T. 10. - №2. - C. 11-18. [Pankratova YuB, Pigarova EA, Dzeranova LK. Vitamin K-dependent proteins: osteocalcin, matrix Gla-protein and extra osseous effects. Obesity and metabolism. 2013;10(2):11-18. (In Russ.)]. doi: https://doi.org/10.14341/2071-8713-4818

11. Khaw K-T, Stewart AW, Waayer D, et al. Effect of monthly high-dose vitamin D supplementation on falls and nonvertebral fractures: secondary and post-hoc outcomes from the randomised, double-blind, placebo-controlled ViDA trial. Lancet Diabetes Endocrinol. 2017;5(6):438-447. doi: https://doi.org/10.1016/S2213-8587(17)30103-1

12. Urayama S, Kawakami A, Nakashima T, et al. Effect of vitamin K2 on osteoblast apoptosis: Vitamin K2 inhibits apoptotic cell death of human osteoblasts induced by Fas, proteasome inhibitor, etoposide, and staurosporine. J Lab Clin Med. 2000;136(3):181-193. doi: https://doi.org/10.1067/mlc.2000.108754

13. Kameda T, Miyazawa K, Mori Y, et al. Vitamin K2 inhibits osteoclastic bone resorption by inducing osteoclast apoptosis. Biochem Biophys Res Commun. 1996;220(3):515-519. doi: https://doi.org/10.1006/bbrc.1996.0436

14. Koshihara Y, Hoshi K, Okawara R, et al. Vitamin K stimulates osteoblastogenesis and inhibits osteoclastogenesis in human bone marrow cell culture. J Endocrinol. 2003;176(3):339-348. doi: https://doi.org/10.1677/joe.0.1760339

15. Ohsaki Y, Shirakawa H, Miura A, et al. Vitamin K suppresses the lipopolysaccharide-induced expression of inflammatory cytokines in cultured macrophage-like cells via the inhibition of the activation of nuclear factor $\mathrm{KB}$ through the repression of IKKa/ $\beta$ phosphorylation. J Nutr Biochem. 2010;21(11):1120-1126. doi: https://doi.org/10.1016/j.jnutbio.2009.09.011

16. Reddi K, Henderson B, Meghji S, et al. Interleukin 6 production by lipopolysaccharide-stimulated human fibroblasts is potently inhibited by naphthoquinone (vitamin K) compounds. Cytokine. 1995;7(3):287-290. doi: https://doi.org/10.1006/cyto.1995.0034
17. Schröder M, Riksen EA, He J, et al. Vitamin K2 Modulates Vitamin D-Induced Mechanical Properties of Human 3D Bone Spheroids In Vitro. JBMR Plus. 2020;4(9):e10394. doi: https://doi.org/10.1002/jbm4.10394

18. Weng SJ, Yan DY, Gu LJ, et al. Combined treatment with vitamin $\mathrm{K} 2$ and PTH enhanced bone formation in ovariectomized rats and increased differentiation of osteoblast in vitro. Chem Biol Interact. 2019;300:101-110. doi: https://doi.org/10.1016/j.cbi.2019.01.012

19. Li H, Zhou Q, Bai BL, et al. Effects of combined human parathyroid hormone (1-34) and menaquinone-4 treatment on the interface of hydroxyapatite-coated titanium implants in the femur of osteoporotic rats. J Bone Miner Metab. 2018;36(6):691-699. doi: https://doi.org/10.1007/s00774-017-0893-9

20. Iwamoto J, Yeh JK, Takeda T. Effect of vitamin K2 on cortical and cancellous bones in orchidectomized and/or sciatic neurectomized rats. J Bone Miner Res. 2003;18(4):776-783. doi: https://doi.org/10.1359/jbmr.2003.18.4.776

21. I wamoto J, Matsumoto H, Tadeda T, et al. Comparison of the effect of vitamin $\mathrm{K}(2)$ and risedronate on trabecular bone in glucocorticoidtreated rats: a bone histomorphometry study [retracted in: Yonsei Med J. 2018;59(1):168]. Yonsei Med J. 2009;50(2):189-194. doi: https://doi.org/10.3349/ymj.2009.50.2.189

22. I wamoto J, Matsumoto H, Takeda T, et al. Effects of vitamin K2 on cortical and cancellous bone mass, cortical osteocyte and lacunar system, and porosity in sciatic neurectomized rats. Calcif Tissue Int. 2010;87(3):254-262. doi: https://doi.org/10.1007/s00223-010-9387-7

23. Kobayashi M, Hara K, Akiyama Y. Effect of menatetrenone (V.K2) on bone mineral density and bone strength in $\mathrm{Ca} / \mathrm{Mg}$ deficient rats. Nihon Yakurigaku Zasshi. 2002;120(3):195-204. doi: https://doi.org/10.1254/fpj.120.195

24. Boulier A, Schwarz J, Lespesailles E, et al. Combination of micellar casein with calcium and vitamins D2 and K2 improves bone status of ovariectomized mice. Osteoporos Int. 2016;27(10):3103-3112. doi: https://doi.org/10.1007/s00198-016-3638-z

25. Gigante A, Torcianti M, Boldrini E, et al. Vitamin K and D association stimulates in vitro osteoblast differentiation of fracture site derived human mesenchymal stem cells. J Biol Regul Homeost Agents. 2008;22(1):35-44

26. Sasaki H, Miyakoshi N, Kasukawa Y, et al. Effects of combination treatment with alendronate and vitamin $\mathrm{K}(2)$ on bone mineral density and strength in ovariectomized mice. J Bone Miner Metab. 2010;28(4):403-409. doi: https://doi.org/10.1007/s00774-009-0148-5

27. Fu X, Moreines J, Booth SL. Vitamin K supplementation does not prevent bone loss in ovariectomized Norway rats. Nutr Metab (Lond). 2012;9(1):12. doi: https://doi.org/10.1186/1743-7075-9-12

28. Kaneki M, Hodges $\mathrm{SJ}$, Hosoi T, et al. Japanese fermented soybean food as the major determinant of the large geographic difference in circulating levels of vitamin K2: possible implications for hip-fracture risk [published correction appears in Nutrition. 2006;22(10):1075. Hedges SJ [corrected to Hodges SJ]]. Nutrition. 2001;17(4):315-321. doi:10.1016/s0899-9007(00)00554-2

29. Iwamoto J. Vitamin $\mathrm{K}_{2}$ therapy for postmenopausal osteoporosis. Nutrients. 2014;6(5):1971-1980. doi: https://doi.org/10.3390/nu6051971

30. Horiuchi T, Kazama H, Araki A, et al. Impaired gamma carboxylation of osteocalcin in elderly women with type II diabetes mellitus: relationship between increase in undercarboxylated osteocalcin levels and low bone mineral density. J Bone Miner Metab. 2004;22(3):236-240. doi: https://doi.org/10.1007/s00774-003-0473-z

31. Knapen MH, Schurgers LJ, Vermeer C. Vitamin K2 supplementation improves hip bone geometry and bone strength indices in postmenopausal women. Osteoporos Int. 2007;18(7):963-972. doi: https://doi.org/10.1007/s00198-007-0337-9

32. Knapen MH, Drummen NE, Smit E, et al. Three-year low-dose menaquinone-7 supplementation helps decrease bone loss in healthy postmenopausal women. Osteoporos Int. 2013;24(9):2499-2507. doi: https://doi.org/10.1007/s00198-013-2325-6

33. Cockayne S, Adamson J, Lanham-New S, et al. Vitamin $K$ and the prevention of fractures: systematic review and meta-analysis of randomized controlled trials [published correction appears in JAMA Intern Med. 2018;178(6):875-876]. Arch Intern Med. 2006;166(12):1256. doi: https://doi.org/10.1001/archinte.166.12.1256 


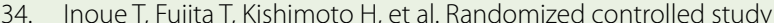
on the prevention of osteoporotic fractures (OF study): a phase IV clinical study of 15-mg menatetrenone capsules. J Bone Miner Metab. 2009;27(1):66-75. doi: https://doi.org/10.1007/s00774-008-0008-8

35. Binkley N, Harke J, Krueger D, et al. Vitamin K treatment reduces undercarboxylated osteocalcin but does not alter bone turnover, density, or geometry in healthy postmenopausal North American women. J Bone Miner Res. 2009;24(6):983-991. doi: https://doi.org/10.1359/jbmr.081254

36. Emaus N, Gjesdal CG, Almås B, et al. Vitamin K2 supplementation does not influence bone loss in early menopausal women: a randomised double-blind placebocontrolled trial. Osteoporos Int. 2010;21(10):1731-1740. doi: https://doi.org/10.1007/s00198-009-1126-4

37. Huang ZB, Wan SL, Lu YJ, et al. Does vitamin K2 play a role in the prevention and treatment of osteoporosis for postmenopausal women: a meta-analysis of randomized controlled trials. Osteoporos Int. 2015;26(3):1175-1186. doi:10.1007/s00198-014-2989-6

38. Tsugawa N, Shiraki M, Suhara Y, et al. Vitamin K status of healthy Japanese women: age-related vitamin $\mathrm{K}$ requirement for gammacarboxylation of osteocalcin. Am J Clin Nutr. 2006;83(2):380-386. doi: https://doi.org/10.1093/ajcn/83.2.380

39. Theuwissen $\mathrm{E}$, Smit $\mathrm{E}$, Vermeer $\mathrm{C}$. The role of vitamin $\mathrm{K}$ in soft-tissue calcification. Adv Nutr. 2012;3(2):166-173. doi: https://doi.org/10.3945/an.111.001628

40. Iwamoto J, Takeda T, Ichimura S. Treatment with vitamin D3 and/or vitamin K2 for postmenopausal osteoporosis. Keio J Med. 2003;52(3):147-150. doi: https://doi.org/10.2302/kjm.52.147

41. Ushiroyama T, Ikeda A, Ueki M. Effect of continuous combined therapy with vitamin $\mathrm{K}(2)$ and vitamin $\mathrm{D}(3)$ on bone mineral density and coagulofibrinolysis function in postmenopausal women. Maturitas. 2002;41(3):211-221. doi: https://doi.org/10.1016/s0378-5122(01)00275-4

42. Tanaka I, Oshima H. Vitamin K2 as a potential therapeutic agent for glucocorticoid -induced osteoporosis. Clinical calcium. 2007;17(11):1738-1744

43. Kanellakis S, Moschonis G, Tenta R, et al. Changes in parameters of bone metabolism in postmenopausal women following a 12-month intervention period using dairy products enriched with calcium, vitamin $\mathrm{D}$, and phylloquinone (vitamin $\mathrm{K}(1)$ ) or menaquinone-7 (vitamin K (2)): the Postmenopausal Health Study II. Calcif Tissue Int. 2012;90(4):251-262 doi: https://doi.org/10.1007/s00223-012-9571-Z

44. Zhang Y, Liu Z, Duan L, et al. Effect of Low-Dose Vitamin K2 Supplementation on Bone Mineral Density in Middle-Aged and Elderly Chinese: A Randomized Controlled Study. Calcif Tissue Int. 2020;106(5):476-485. doi: https://doi.org/10.1007/s00223-020-00669-4

45. Suzuki K, Tsuji S, Fukushima Y, et al. Clinical results of alendronate monotherapy and combined therapy with menatetrenone ( $\left.\mathrm{VitK}_{2}\right)$ in postmenopausal RA patients. Mod Rheumatol. 2013;23(3):450-455 doi: https://doi.org/10.1007/s10165-012-0678-x

46. Tanaka S, Miyazaki T, Uemura Y, et al. Comparison of concurrent treatment with vitamin $\mathrm{K}_{2}$ and risedronate compared with treatment with risedronate alone in patients with osteoporosis: Japanese Osteoporosis Intervention Trial-03. J Bone Miner Metab. 2017;35(4):385-395. doi: https://doi.org/10.1007/s00774-016-0768-5

47. Jiang $N$, Xia W. Assessment of bone quality in patients with diabetes mellitus. Osteoporos Int. 2018;29(8):1721-1736. doi: https://doi.org/10.1007/s00198-018-4532-7

48. Shah VN, Carpenter RD, Ferguson VL, Schwartz AV. Bone health in type 1 diabetes. Curr Opin Endocrinol Diabetes Obes. 2018;25(4):231-236. doi: https://doi.org/10.1097/MED.0000000000000421

49. Hamilton EJ, Rakic V, Davis WA, et al. Prevalence and predictors of osteopenia and osteoporosis in adults with Type 1 diabetes. Diabet Med. 2009;26(1):45-52 doi: https://doi.org/10.1111/j.1464-5491.2008.02608.x

50. Maggio $A B$, Ferrari $S$, Kraenzlin $M$, et al. Decreased bone turnover in children and adolescents with well controlled type 1 diabetes. J Pediatr Endocrinol Metab. 2010;23(7):697-707. doi: https://doi.org/10.1515/jpem.2010.23.7.697

51. Bonds DE, Larson JC, Schwartz AV, et al. Risk of fracture in women with type 2 diabetes: the Women's Health Initiative Observational Study. J Clin Endocrinol Metab. 2006;91(9):3404-3410. doi: https://doi.org/10.1210/jc.2006-0614
52. Sundararaghavan V, Mazur MM, Evans B, Liu J, Ebraheim NA. Diabetes and bone health: latest evidence and clinical implications. Ther Adv Musculoskelet Dis. 2017:9(3):67-74 doi: https://doi.org/10.1177/1759720X16687480

53. Zhukouskaya WV, Eller-Vainicher C, Gaudio A, et al. The utility of lumbar spine trabecular bone score and femoral neck bone mineral density for identifying asymptomatic vertebral fractures in well-compensated type 2 diabetic patients [published correction appears in Osteoporos Int. 2016;27(1):421. Ellen-Vainicher C [Corrected to Eller-Vainicher C]]. Osteoporos Int. 2016;27(1):49-56. doi: https://doi.org/10.1007/s00198-015-3212-0

54. Vestergaard P. Discrepancies in bone mineral density and fracture risk in patients with type 1 and type 2 diabetes — a meta-analysis. Osteoporos Int. 2007;18(4):427-444. doi: https://doi.org/10.1007/s00198-006-0253-4

55. Neumann T, Lodes S, Kästner B, et al. Trabecular bone score in type 1 diabetes--a cross-sectional study. Osteoporos Int. 2016;27(1):127-133. doi: https://doi.org/10.1007/s00198-015-3222-y

56. Kim JH, Choi HJ, Ku EJ, et al. Trabecular bone score as an indicator for skeletal deterioration in diabetes. J Clin Endocrinol Metab. 2015;100(2):475-482. doi: https://doi.org/10.1210/jc.2014-2047

57. McCloskey EV, Odén A, Harvey NC, et al. A Meta-Analysis of Trabecular Bone Score in Fracture Risk Prediction and Its Relationship to FRAX. J Bone Miner Res. 2016;31(5):940-948. doi: https://doi.org/10.1002/jbmr.2734

58. Napoli N, Chandran M, Pierroz DD, et al. Mechanisms of diabetes mellitus-induced bone fragility. Nat Rev Endocrinol. 2017;13(4):208-219. doi: https://doi.org/10.1038/nrendo.2016.153

59. Федорова О.С., Гурьева И.В., Строков И.А. Влияние дистальной полинейропатии на нарушение равновесия у больных сахарным диабетом // Медииинский Совет. - 2013. — №12. C. 118-119. [Fedorova OS, Gurieva IV, Strokov IA. The effect of distal polyneuropathy on balance disorder in patients with diabetes. Medical Council. 2013;(12):118-119. (In Russ.)]. doi: https://doi.org/10.21518/2079-701X-2013-12-118-119

60. Kalaitzoglou E, Popescu I, Bunn RC, et al. Effects of Type 1 Diabetes on Osteoblasts, Osteocytes, and Osteoclasts. Curr Osteoporos Rep. 2016;14(6):310-319. doi: https://doi.org/10.1007/s1 1914-016-0329-9

61. Kierdorf K, Fritz G. RAGE regulation and signaling in inflammation and beyond. J Leukoc Biol. 2013;94(1):55-68. doi: https://doi.org/10.1189/jlb.1012519

62. Aikawa E, Fujita R, Asai M, et al. Receptor for Advanced Glycation End Products-Mediated Signaling Impairs the Maintenance of Bone Marrow Mesenchymal Stromal Cells in Diabetic Model Mice. Stem Cells Dev. 2016;25(22):1721-1732. doi: https://doi.org/10.1089/scd.2016.0067

63. Lewiecki EM. Role of sclerostin in bone and cartilage and its potential as a therapeutic target in bone diseases. Ther Adv Musculoskelet Dis. 2014;6(2):48-57. doi: https://doi.org/10.1177/1759720X13510479

64. Wędrychowicz A, Sztefko K, Starzyk JB. Sclerostin and its significance for children and adolescents with type 1 diabetes mellitus (T1D). Bone. 2019;120:387-392. doi: https://doi.org/10.1016/j.bone.2018.08.007

65. Yamamoto M, Yamauchi M, Sugimoto T. Elevated sclerostin levels are associated with vertebral fractures in patients with type 2 diabetes mellitus. J Clin Endocrinol Metab. 2013:98(10):4030-4037. doi: https://doi.org/10.1210/jc.2013-2143

66. Rubin MR, Patsch JM. Assessment of bone turnover and bone quality in type 2 diabetic bone disease: current concepts and future directions. Bone Res. 2016;4(1):16001. doi: https://doi.org/10.1038/boneres.2016.1

67. Starup-Linde J, Eriksen SA, Lykkeboe S, et al. Biochemical markers of bone turnover in diabetes patients--a metaanalysis, and a methodological study on the effects of glucose on bone markers. Osteoporos Int. 2014;25(6):1697-1708. doi: https://doi.org/10.1007/s00198-014-2676-7

68. Bone HG, Lindsay R, McClung MR, et al. Effects of pioglitazone on bone in postmenopausal women with impaired fasting glucose or impaired glucose tolerance: a randomized, double-blind, placebocontrolled study. J Clin Endocrinol Metab. 2013;98(12):4691-4701. doi: https://doi.org/10.1210/jc.2012-4096

69. Watts NB, Bilezikian JP, Usiskin K, et al. Effects of Canagliflozin on Fracture Risk in Patients With Type 2 Diabetes Mellitus. J Clin Endocrinol Metab. 2016;101(1):157-166. doi: https://doi.org/10.1210/jc.2015-3167 
70. Tang HL, Li DD, Zhang JJ, et al. Lack of evidence for a harmful effect of sodium-glucose co-transporter 2 (SGLT2) inhibitors on fracture risk among type 2 diabetes patients: a network and cumulative meta-analysis of randomized controlled trials. Diabetes Obes Metab. 2016;18(12):1199-1206. doi: https://doi.org/10.1111/dom.12742

71. Лебедев Д.А., Андреева А.Т., Кокина М.А., и др. Влияние терапии ингибитором натрий-глюкозного ко-транспортера 2 типа на фосфорно-кальциевый обмен у пациентов сахарным диабетом 2 типа // Ocmeonopoз и остеonamuи. — 2020. - T. 23. — №2. C. 84. [Lebedev DA, Andreeva AT, Kokina MA, et al. The influence of treatment with the sodium-glucose co-transporter type 2 inhibitors for phosphorus-calcium exchange inpatients with type 2 diabetes. Osteoporosis and Bone Diseases. 2020;23(2):84. (In Russ.)].

72. Li Y, Chen JP, Duan L, Li S. Effect of vitamin K2 on type 2 diabetes mellitus: A review. Diabetes Res Clin Pract. 2018;136:39-51. doi: https://doi.org/10.1016/j.diabres.2017.11.020

73. Yasuda S, Wada S. Diabetic osteopahty and vitamin K. Clin Calcium. 2006;16(8):1351-57. doi: https://doi.org/CliCa060813511357

74. Poon CC, Li RW, Seto SW, et al. In vitro vitamin K(2) and 1a,25dihydroxyvitamin $\mathrm{D}(3)$ combination enhances osteoblasts anabolism of diabetic mice. Eur J Pharmacol. 2015:767:30-40 doi: https://doi.org/10.1016/j.jphar.2015.09.048

75. Iwamoto J, Seki A, Sato Y, et al. Vitamin $K_{2}$ prevents hyperglycemia and cancellous osteopenia in rats with streptozotocininduced type 1 diabetes. Calcif Tissue Int. 2011;88(2):162-168. doi: https://doi.org/10.1007/s00223-010-9441-5

76. Horiuchi T, Kazama H, Araki A, et al. Impaired gamma carboxylation of osteocalcin in elderly women with type Il diabetes mellitus: relationship between increase in undercarboxylated osteocalcin levels and low bone mineral density. J Bone Miner Metab. 2004;22(3):236-240. doi: https://doi.org/10.1007/s00774-003-0473-z

77. Aguayo-Ruiz Jl, García-Cobián TA, Pascoe-González S, et al. Effect of supplementation with vitamins D3 and K2 on undercarboxylated osteocalcin and insulin serum levels in patients with type 2 diabetes mellitus: a randomized, double-blind, clinical trial. Diabetol Metab Syndr. 2020;12:73. doi: https://doi.org/10.1186/s13098-020-00580-w

78. Bartstra JW, Draaisma F, Zwakenberg SR, et al. Six months vitamin $\mathrm{K}$ treatment does not affect systemic arterial calcification or bone mineral density in diabetes mellitus 2. Eur J Nutr. 2021;60(3):16911699. doi: https://doi.org/10.1007/s00394-020-02412-z

\section{ИНФОРМАЦИЯ ОБ АВТОРАХ [AUTHORS INFO]}

*Каронова Татьяна Леонидовна, д.м.н., главный научный сотрудник НИЛ клинической эндокринологии Института эндокринологии, профессор кафедры эндокринологии ФГБУ «Национальный медицинский исследовательский центр им. В.А. Алмазова» МЗ РФ [Tatiana L. Karonova, MD, PhD, chief researcher of Clinical Endocrinology Laboratory of the Institute of Endocrinology, professor of the Department of Endocrinology, Almazov National Medical Research Centre of the Ministry of Healthcare of Russia]; адрес: 194021, Санкт-Петербург, пр. Пархоменко, д. 15 [аddress: 194021 , Saint-Petersburg, 15 Parkhomenko Avenue]; ORCID: https://orcid.org/0000-0002-1547-0123; eLibrary SPIN: 3337-4071; e-mail: karonova@mail.ru

Радугин Федор Михайлович, аспирант кафедры эндокринологии ФГБУ «Национальный медицинский исследовательский центр им. В.А. Алмазова» МЗ РФ [Fyodor M. Radugin, postgraduate student of the Department of Endocrinology, Almazov National Medical Research Centre of the Ministry of Healthcare of Russia]; ORCID: https://orcid.org/0000-0003-3224-1573; eLibrary SPIN: 7043-3620; e-mail: radugin.f.m@gmail.com

\section{ИНФОРМАЦИЯ}

Рукопись получена: 13.07.2021. Одобрена к публикации: 18.10.2021.

\section{ЦИТИРОВАТЬ:}

Радугин Ф.М., Каронова Т.Л. Особенности костного ремоделирования у больных сахарным диабетом: фокус на вита-

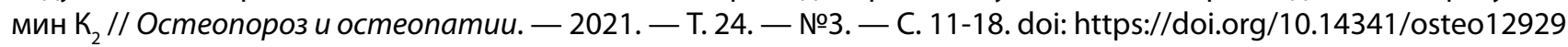

\section{TO CITE THIS ARTICLE:}

Radugin FM, Karonova TL. Bone metabolism in diabetes mellitus: focus on vitamin $\mathrm{K}_{2}$. Osteoporosis and bone diseases. 2021;24(3):11-18. doi: https://doi.org/10.14341/osteo12929 